\title{
Remarks on the geometry of the feedback loop
}

\author{
Z. Szabó and J. Bokor
}

\begin{abstract}
Youla parametrization of stabilizing controllers is a fundamental result of control theory. It provides a theoretical and practical tool that renders LFT loops into model matching framework. Based on the geometric techniques introduced in our previous work we have provided a controller blending rule that defines a controller semigroup structure based only on the knowledge of the plant and a single stabilizing controller and we have introduced a novel, geometry based parametrization. In this paper we investigate the possibility to construct stabilizing fractions of the given stabilizing controller relative to the blending rule. We also extend our geometry based framework to the LFT loops. Our main goal is to show that every controller which stabilizes the interior loop also stabilizes the LFT loop. Contrary to the expectations, this problem is far from being trivial.
\end{abstract}

\section{INTRODUCTION AND MOTIVATION}

Klein proposed group theory as a mean of formulating and understanding geometrical constructions. In [8] the authors emphasise Klein's approach to geometry and demonstrate that a natural framework to formulate various control problems is the world that contains as points equivalence classes determined by stabilizable plants and whose natural motions are the Möbius transforms. The observation that any geometric property of a configuration, which is invariant under an euclidean or hyperbolic motion, may be reliably investigated after the data has been moved into a convenient position in the model, facilitates considerably the solution of the problems. In [9], [10], [11], as a main contribution relative to the previous efforts it is shown that, in contrast to the classical Youla approach, there is a parametrisation of the entire controller set which can be described entirely in a coordinate free way, i.e., just by using the knowledge of the plant $P$ and of the given stabilizing controller $K_{0}$. The corresponding parameter set is given in geometric terms, i.e., by providing an associated algebraic (semigroup, group) structure. Moreover, it turns out that the geometry of stable controllers is surprisingly simple.

The first part of this paper extends these results by investigating the problem of halving a stabilizing controller in the sense of the blending operation, keeping the stabilizing property invariant. In general, we call these fractional controllers, as they are analogous to the fractions of the integer numbers.

The second part of the paper extends the geometric techniques to the LFT framework, showing that the already introduced blending operator still works in this context,

Institute for Computer Science and Control, Hungarian Academy of Sciences, Hungary, (Tel: +36-1-279-6171; e-mail: szabo.zoltan@sztaki.mta.hu). This work has been supported by the GINOP-2.3.2-15-2016-00002 grant of the Ministry of National Economy of Hungary and by the European Commission through the H2020 project EPIC under grant No. 739592. too. The classical Youla approach that students learn for rational LTI systems provides an easy way to relate the LFT loop with the model matching framework defined entirely on the set of stable systems. This result, however, actually has two parts: the qualitative message, i.e., every stabilizing controller of the interior part stabilizes the LFT loop and the parametrization dependent part, which is actually the model matching form.

In our context the first part is relevant: contrary to the expectations, this problem is far from being trivial in its generality. Despite of the fact that the geometrical structure of the controller set suggests that the assertion is always true, in this paper we can provide a general proof only for the case, when a double coprime factorization of the interior part exists.

Section II gives the basic notions related to feedback and LFT stability and recalls the fundamental result of the Youla parametrization. Section III recalls some previous results of the authors: a natural blending method is introduced that acts directly on the controllers and keeps stability of the loop. It also provides a geometric based parametrization of the stabilizing controllers by showing how the geometric view can be applied to reveal the coordinate free nature of the parametrization. In Section IV we investigate conditions for the existence of the halved controllers. Section V is dedicated to the geometry of the LFT controllers. Finally some conclusions are formulated.

\section{BASIC SETTINGS}

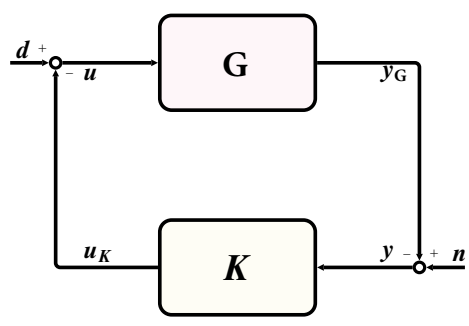

Fig. 1. Feedback connection

To fix the ideas let us consider the feedback-connection depicted on Figure 1. It is convenient to consider the signals

$$
w=\left(\begin{array}{l}
d \\
n
\end{array}\right), g=\left(\begin{array}{c}
u \\
y_{G}
\end{array}\right), k=\left(\begin{array}{c}
u_{K} \\
y
\end{array}\right), z=\left(\begin{array}{l}
u \\
y
\end{array}\right) \in \mathcal{H},
$$

where $\mathcal{H}=\mathcal{H}_{1} \oplus \mathcal{H}_{2}$ and we suppose that the signals are elements of the Hilbert space $\mathcal{H}_{1}, \mathcal{H}_{2}$ (e.g., $\mathcal{H}_{i}=\mathcal{L}^{n_{i}}[0, \infty)$ ) endowed by a resolution structure which determines the 
causality concept on these spaces. In this model the plant $G$ and the controller $K$ are linear causal maps. For more details on this general setting, see [3].

The feedback connection is called well-posed if for every $w \in \mathcal{H}$ there is a unique $g$ and $k$ such that $w=g+k$ (causal invertibility) and the pair $(G, K)$ is called stable if the map $w \rightarrow z$ is a bounded causal map, i.e., the pair $(G, K)$ is called well-posed if the inverse

$$
\begin{aligned}
\mathcal{H}(G, K) & =\left(\begin{array}{cc}
I & K \\
G & I
\end{array}\right)^{-1}=\left(\begin{array}{cc}
S_{u} & S_{c} \\
S_{g} & S_{y}
\end{array}\right)= \\
& =\left(\begin{array}{cc}
(I-K G)^{-1} & -K(I-G K)^{-1} \\
-G(I-K G)^{-1} & (I-G K)^{-1}
\end{array}\right)
\end{aligned}
$$

exists (causal invertibility), and it is called stable if all the block elements are stable.

The lower and an upper LFT is defined as

$$
\mathfrak{F}_{l}(P, K)=P_{z w}+P_{z u} K\left(I-P_{y u} K\right)^{-1} P_{y w}
$$

and

$$
\mathfrak{F}_{u}(P, \Delta)=P_{y u}+P_{y w} \Delta\left(I-P_{z w} \Delta\right)^{-1} P_{z u} .
$$

Stability of the LFT loop means that the causal map $\mathcal{L}(P, K)$ that relates the signals $(z, u, y)$ to $(w, d, n)$ is invertible and the inverse map is stable, see Figure 2(a). It turns out that this is equivalent to the stability of the extended feedback loop for $\Delta_{p}=0_{p}$, see Figure 2(b).

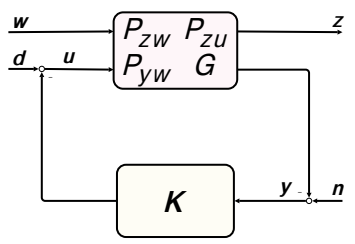

(a) Performance

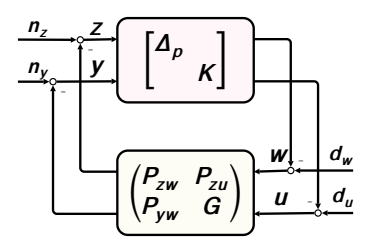

(b) Stability
Fig. 2. Stability of LFTs

Starting from the basic relation between the relevant signals we have

$$
\left(\begin{array}{l}
d_{u} \\
n_{y} \\
d_{w} \\
n_{z}
\end{array}\right)=\left(\begin{array}{cccc}
I_{u} & K & 0 & 0 \\
G & I_{y} & P_{y w} & 0 \\
0 & 0 & I_{w} & \Delta_{p} \\
P_{z u} & 0 & P_{z w} & I_{z}
\end{array}\right)\left(\begin{array}{c}
u \\
y \\
w \\
z
\end{array}\right) .
$$

Invertibility of the operator is equivalent to the nonsingularity of the corresponding Schur complement, i.e, nonsingularity of

$$
\begin{aligned}
& \left(\begin{array}{cc}
I_{w} & \Delta_{p} \\
P_{z w} & I_{z}
\end{array}\right)-\left(\begin{array}{cc}
0 & 0 \\
P_{z u} & 0
\end{array}\right) \mathcal{H}(G, K)\left(\begin{array}{cc}
0 & 0 \\
P_{y w} & 0
\end{array}\right)= \\
& =\left(\begin{array}{cc}
I_{w} & \Delta_{p} \\
\mathfrak{F}_{l}(P, K) & I_{z}
\end{array}\right),
\end{aligned}
$$

which is always fulfilled for $\Delta_{p}=0_{p}$, when the inverse is

$$
\begin{aligned}
& \left(\begin{array}{cc}
\mathcal{H}(G, K) & -\mathcal{H}(G, K)\left(\begin{array}{cc}
0 & 0 \\
P_{y w} & 0
\end{array}\right) \\
-\left(\begin{array}{cc}
0 & 0 \\
P_{z u} & 0
\end{array}\right) \mathcal{H}(G, K) & \left(\begin{array}{cc}
I_{w} & 0 \\
-\mathfrak{F}_{l}(P, K) & I_{z}
\end{array}\right)
\end{array}\right) \\
& =\left(\begin{array}{cc}
\mathcal{H}(G, K) & \left(\begin{array}{cc}
-S_{c} P_{y w} & 0 \\
-S_{y} P_{y w} & 0
\end{array}\right) \\
0 & 0 \\
I_{w} & 0 \\
-P_{z u} S_{u} & -P_{z u} S_{c}
\end{array}\right) .
\end{aligned}
$$

On the other hand one has

$$
\left(\begin{array}{ccc}
I_{w} & 0 & 0 \\
0 & I_{u} & 0 \\
-P_{y w} & 0 & I_{y}
\end{array}\right)\left(\begin{array}{l}
w \\
d \\
n
\end{array}\right)=\left(\begin{array}{ccc}
I_{z} & -P_{z u} & 0 \\
0 & I_{u} & K \\
0 & G & I_{y}
\end{array}\right)\left(\begin{array}{l}
z \\
u \\
y
\end{array}\right)
$$

Thus a routine computation reveals that stability of the LFT loop is equivalent to the stability of the extended feedback loop with $\Delta_{p}=0_{p}$, as it was claimed.

As a consequence, stability questions of LFT loops can be reduced to the investigation of the configuration determined by $(P, \operatorname{diag}(0, K))$. It is obvious that the LFT loop is welldefined if and only if $(G, K)$ is well defined. However, it is less obvious whether this claim remains true for stability.

\section{A. Youla parametrization}

A fundamental result concerning feedback stabilization of the connection on Figure 1 is the description of the set of the stabilizing controllers. A standard assumption is that among the stable factorizations there exists a special one, called double coprime factorization, i.e., $G=N M^{-1}=\tilde{M}^{-1} \tilde{N}$ and there are causal bounded systems $U, V, \tilde{U}$ and $\tilde{V}$, with invertible $V$ and $\tilde{V}$, such that

$$
\left(\begin{array}{cc}
\tilde{V} & -\tilde{U} \\
-\tilde{N} & \tilde{M}
\end{array}\right)\left(\begin{array}{cc}
M & U \\
N & V
\end{array}\right)=\tilde{\Sigma}_{G} \Sigma_{G}=\left(\begin{array}{cc}
I & 0 \\
0 & I
\end{array}\right),
$$

an assumption which is often made when setting the stabilization problem, [15], [3]. The existence of a double coprime factorization implies feedback stabilizability, actually $K_{0}=$ $U V^{-1}=\tilde{V}^{-1} \tilde{U}$ is a stabilizing controller. In most of the usual model classes actually there is an equivalence.

For a fixed plant $G$ let us denote by $\mathbb{W}_{G}$ the set of well-posed controllers, while $\mathbb{G}_{G} \subset \mathbb{W}_{G}$ denotes the set of stabilizing controllers.

Given a double coprime factorization the set of the stabilizing controllers is provided through the well-known Youla parametrization, [7], [14]:

$$
\mathbb{G}_{G}=\left\{K=\mathfrak{M}_{\Sigma_{G}}(Q) \mid Q \in \mathbb{Q}_{\Sigma}\right\},
$$

where $\mathbb{Q}_{\Sigma}=\left\{Q \mid Q\right.$ stable,$(V+N Q)^{-1}$ exists $\}$ and

$$
\mathfrak{M}_{\Sigma_{G}}(Q)=(U+M Q)(V+N Q)^{-1} .
$$

For a recent work that covers most of the known control system methodologies using a unified approach based on the Youla parameterization, see [5]. Note, that $\mathbb{Q}_{I}=\mathbb{Q}$ is the set of stable systems. Here $\mathfrak{M}_{T}(Z)$ is the Möbius transformation corresponding to the symbol $T$ :

$$
\mathfrak{M}_{T}(Z)=(B+A Z)(D+C Z)^{-1}, \text { with } T=\left(\begin{array}{ll}
A & B \\
C & D
\end{array}\right),
$$


on the domain $\operatorname{dom}_{\mathfrak{M}_{T}}=\left\{Z \mid(D+C Z)^{-1}\right.$ exists $\}$. Note that

$$
Q_{K}=\mathfrak{M}_{\tilde{\Sigma}_{G}}(K)=(\tilde{V} K-\tilde{U})(\tilde{M}-\tilde{N} K)^{-1},
$$

and thus $Q=0_{K}$ corresponds to $K_{0}=U V^{-1}$.

Since the dimensions of the controller and plant are different, it is convenient to distinguish the zero controller and zero plant by an index, i.e., $0_{K}$ and $0_{G}$, respectively.

Finally, note, that the entire construction has a considerable freedom in the choice of the given elements, like $\Sigma_{P}$ and $Q$, which makes possible to embed a given system in different frameworks. The standard example is to let the parameter $Q$ to be a stable rational LTI system. However, one can consider it as a stable linear parameter varying (LPV) or even switched system obtaining an LPV controller. But nothing prevents us to set also $M$ and $N$ to be LPV systems even the original system was an LTI one, see, e.g., [12].

\section{GROUP OF CONTROLLERS}

It is obvious that in the particular case when $G=0_{G}$ we have $\mathbb{G}_{G}=\mathbb{Q}$, i.e., mere addition preserves well-posedness and stability. Moreover, the set of these controllers forms the usual additive group $(\mathbb{Q},+)$ with neutral element $0_{K}$ and inverse element $Q \rightarrow-Q$. In the general case, however, addition of controllers neither ensure well-posedness nor stability.

The most straightforward approach to obtain a stability preserving operation is to find a suitable parametrization of the stabilizing controllers, where the parameter space possesses a blending operation. As an example for this indirect ( Youla based) blending is provided by the Youla parametrization. However, this mere addition on the Youla parameter level does not lead, in general, to a "simple" operation on the level of controllers:

$$
K=\mathfrak{M}_{\Sigma_{G}}\left(\left(\mathfrak{M}_{\tilde{\Sigma}_{G}}\left(K_{1}\right)+\mathfrak{M}_{\tilde{\Sigma}_{G}}\left(K_{2}\right)\right)\right) .
$$

The unit element of this operation is the controller $K_{0}$ which defines $\Sigma_{G}$. Note that an obstruction might appear if the sum of the Youla parameters are not in the domain of $\mathfrak{M}_{\Sigma_{G}}$, e.g., for non strictly proper plants where some of the non strictly proper parameters are out-ruled.

\section{A. Direct blending}

The observation that

$$
\left(\begin{array}{cc}
I & K \\
G & I
\end{array}\right)=\left(\begin{array}{cc}
I & 0 \\
G & I
\end{array}\right)\left(\begin{array}{cc}
I & K_{1} \\
0 & I-G K_{1}
\end{array}\right)\left(\begin{array}{cc}
I & K_{2} \\
0 & I-G K_{2}
\end{array}\right) .
$$

leads to operation

$$
K=K_{1}\left(I-G K_{2}\right)+K_{2}=K_{1} \sqcup_{G} K_{2},
$$

under which well-posed controllers form a group $\left(\mathbb{W}_{G}, \square_{G}\right)$. The unit of this group is the zero controller $K=0_{K}$ and the corresponding inverse elements are given by

$$
K^{\boxminus_{G}}=-K(I-G K)^{-1} .
$$

Note that

$$
I-G K^{\boxminus_{G}}=(I-G K)^{-1} .
$$

Clearly not all elements of $\mathbb{W}_{G}$ are stabilizing, e.g., $0_{K}$ is not stabilizing for an unstable plant.

Theorem 1: $\left(\mathbb{G}_{G}, \sqcup_{G}\right)$ with the operation (blending) defined in (8) is a semigroup.

Note, that

$$
(I-G K)^{-1}=\left(I-G K_{2}\right)^{-1}\left(I-G K_{1}\right)^{-1} .
$$

By using the notation

$$
\left(\begin{array}{cc}
I & K \\
G & I
\end{array}\right)=\left(\begin{array}{cc}
I & 0 \\
G & I
\end{array}\right)\left(\begin{array}{cc}
I & K \\
0 & I-G K
\end{array}\right)=R_{G} T_{K}^{(G)}
$$

we have the group homomorphism $T_{K_{1}}^{(G)} T_{K_{2}}^{(G)}=T_{K_{1} \square_{G} K_{2}}^{(G)}$

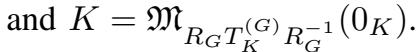

As a final remark observe that the blending (8) is multiplicative, as the notation suggests. It is a routine calculation to show that the blending of the inverses is related to the original blending as:

$$
K=K_{1} \unlhd_{G} K_{2} \quad \text { iff } \quad K^{\boxminus_{G}}=K_{2}^{\boxminus_{G}} \unlhd_{G} K_{1}^{\boxminus_{G}} .
$$

\section{B. Strong stability}

The semigroup $\left(\mathbb{G}_{G}, \ominus_{G}\right)$ does not have a unit, in general. However, if there is a stabilizing controller $K_{0}$ such that

$$
K_{0}^{\boxminus_{G}}=-K_{0}\left(I-G K_{0}\right)^{-1}
$$

is also a stabilizing controller, i.e., $K_{0}$ is stable, then $\left(\mathbb{G}_{G}, \otimes_{G}\right)$ with

$$
K_{1} \otimes_{G} K_{2}=K_{1} \unrhd_{G} K_{0}^{\ominus_{G}} \unlhd_{G} K_{2}
$$

is a semigroup with a unit $\left(K_{0}\right)$. This may happen only if the plant is strongly stabilizable.

If we denote by $\mathbb{S}_{G}$ the set of strongly stabilising controllers, then if this set is not empty, then

Theorem 2: $\left(\mathbb{S}_{G}, \bigotimes_{G}\right)$ with the operation (blending) defined as

$$
\begin{aligned}
& K=K_{1} \otimes_{G} K_{2}=K_{1} \unrhd_{G} K_{0}^{\boxminus_{G}} \unrhd_{G} K_{2}= \\
& =K_{2}+\left(K_{1}-K_{0}\right)\left(I-G K_{0}\right)^{-1}\left(I-G K_{2}\right)
\end{aligned}
$$

is the group of strongly stable controllers, where $K_{0} \in \mathbb{S}_{G}$ is arbitrary. The corresponding inverse is given by

$$
K^{\otimes_{G}^{-1}}=K_{0}-\left(K-K_{0}\right)(I-G K)^{-1}\left(I-G K_{0}\right) .
$$

At this point recall that the necessary and sufficient conditions for the existence of a strongly stabilizing controller for a finite rank LTI plant $G$ can be formulated in terms of the parity interlacing property: there exists a strongly stabilizing controller if and only if the number of poles of $G$ (counted according to their McMillan degrees) between every pair of real blocking zeros of $G$ in the extended right half plane is even, [13]. However, if $G$ is an LTV plant that is internally stabilizable, then it can be internally stabilized by a stable LTV controller, see [6]. Thus, in the general context of this paper it can be assumed the existence of such controllers, i.e., $\mathbb{S}_{G}$ is not void. However, note that even 
if it exists, construction of a stable controller might be a nontrivial task, in general. In practical applications strongly stabilizing controllers are preferred, see [4].

As a final remark, observe that

$$
\left(\begin{array}{cc}
I & -K_{0} \\
S_{g} & S_{y}
\end{array}\right)\left(\begin{array}{cc}
S_{u} & K_{0} \\
-S_{g} & I
\end{array}\right)=\left(\begin{array}{cc}
I & 0 \\
0 & I
\end{array}\right)
$$

i.e., if $K_{0}$ is stable, then we have a granted double coprime factorization given in terms of the original data.

\section{A coordinate free parametrization}

Let us fix a stabilizing controller, say $K_{0}$. Based on the Youla parametrization one can obtain the formulae

$$
\begin{aligned}
& K=\mathfrak{M}_{\Gamma_{G, K_{0}}}(R)=\mathfrak{F}_{l}\left(\Psi_{G, K_{0}}, R\right), \\
& R=\mathfrak{M}_{\Gamma_{G, K_{0}}^{-1}}(K)=\mathfrak{F}_{l}\left(\Phi_{G, K_{0}}, K\right),
\end{aligned}
$$

with

$$
\begin{aligned}
& \Gamma_{G, K_{0}}=\left(\begin{array}{cc}
S_{u} & K_{0} \\
-S_{g} & I
\end{array}\right), \quad \Psi_{G, K_{0}}=\left(\begin{array}{cc}
K_{0} & I \\
I & S_{g}
\end{array}\right), \quad(18) \\
& \Gamma_{G, K_{0}}^{-1}=\left(\begin{array}{cc}
I & -K_{0} \\
S_{g} & S_{y}
\end{array}\right), \Phi_{G, K_{0}}=\left(\begin{array}{cc}
-K_{0} S_{y}^{-1} & S_{u}^{-1} \\
S_{y}^{-1} & G
\end{array}\right),
\end{aligned}
$$

where

$$
R \in \mathbb{R}_{K_{0}}^{Y}=\left\{\tilde{V}^{-1} Q V^{-1} \mid Q \in \mathbb{Q}\right\} .
$$

Observe, however, that (17) is defined exactly on $\mathbb{W}_{G}$ and let the restriction on the stabilizing controllers be denoted by $\mathbb{R}_{K_{0}}=\left\{\mathfrak{F}_{l}\left(\Phi_{G, K_{0}}, K\right) \mid K \in \mathbb{G}_{G}\right\}$. Actually the set $\mathbb{R}_{K_{0}}=\mathbb{R}_{K_{0}}^{Y}$ does not depend on any special factorization. It can be obtained directly, i.e., without any reference to some particular factorization of the plant or of the controller, starting from

$$
\left(\begin{array}{cc}
I & K \\
G & I
\end{array}\right)=\left(\begin{array}{cc}
I & K_{0} \\
G & I
\end{array}\right)+\left(\begin{array}{l}
I \\
0
\end{array}\right)\left(\begin{array}{ll}
K-K_{0}
\end{array}\right)\left(\begin{array}{ll}
0 & I
\end{array}\right)
$$

and applying two times the matrix inversion lemma to obtain first

$$
\left(\begin{array}{cc}
I & K \\
G & I
\end{array}\right)^{-1}=\left(\begin{array}{cc}
I & K_{0} \\
G & I
\end{array}\right)^{-1}-\left(\begin{array}{c}
S_{u} \\
S_{g}
\end{array}\right) R\left(\begin{array}{ll}
S_{g} & S_{y}
\end{array}\right),
$$

with $R=\left(K-K_{0}\right)\left(I+S_{g}\left(K-K_{0}\right)\right)^{-1}$ and then

$$
\left(\begin{array}{cc}
I & K \\
G & I
\end{array}\right)=\left(\begin{array}{cc}
I & K_{0} \\
G & I
\end{array}\right)+\left(\begin{array}{l}
I \\
0
\end{array}\right) R\left(I-S_{g} R\right)^{-1}\left(\begin{array}{ll}
0 & I
\end{array}\right) .
$$

This is the point where the geometric view and the coordinate free results can be applied. On $\mathbb{R}_{K_{0}}$ we have the blending rule

$$
R_{2} \odot_{G, K_{0}} R_{1}=K_{0}+S_{u} R_{1}+R_{2} S_{y}-R_{2} S_{y} S_{g} R_{1} .
$$

For the stable controllers the parameter blending is more simple:

$$
\begin{aligned}
& R_{2} \otimes_{G, K_{0}} R_{1}=R_{2}+R_{1}-R_{2} S_{g} R_{1}, \\
& R^{\otimes_{G, K_{0}}^{-1}}=-R\left(I-S_{g} R\right)^{-1} .
\end{aligned}
$$

One can observe that we have

$$
\mathbb{Q}_{\Sigma}=\left\{Q \mid Q \text { stable, }\left(I-S_{g} Q\right)^{-1} \text { exists }\right\},
$$

i.e., $\mathbb{Q}_{\Sigma}$ is representation independent. Moreover, from (21) it is easy to see that besides $K_{0} \in \mathbb{R}_{K_{0}}$, the inclusion $\mathbb{Q}_{\Sigma} \subset$ $\mathbb{R}_{K_{0}}$ also holds, i.e., we know by start significant, nontrivial part of $\mathbb{R}_{K_{0}}$.

\section{FRACTIONAL CONTROLLERS}

Given a stabilizing controller $K_{0}$ it is a natural question whether there exists a stabilizing controller $X$ such that

$$
K_{0}=X \bullet_{G} X=[2 X]_{\square_{G}},
$$

i.e., $X=\left[\frac{1}{2} K_{0}\right]_{\varpi_{G}}$. In general, to have $K_{0}=[k X]_{\square_{G}}$, i.e., $X=\left[\frac{1}{k} K_{0}\right]_{\square_{G}}$, respectively. The existence of such a controller might made possible to have controllers of type $K=\left[\left(m+\frac{l}{k}\right) X\right]_{\square_{G}}$.

From (26) it follows that

$K_{0}=X\left(I+S_{y, X}\right) S_{y, X}^{-1}$, i.e., $X=K_{0} S_{y, X}\left(I+S_{y, X}\right)^{-1}$,

with $S_{y, X}=(I-G X)^{-1}$, provided that the corresponding inverses exist. Recall that $S_{y}=S_{y, X}^{2}$. Thus

$$
X=K_{0} S_{y}^{1 / 2}\left(I+S_{y}^{1 / 2}\right)^{-1}=K_{0}-K_{0}\left(I+S_{y}^{1 / 2}\right)^{-1} .
$$

Analogously, one can deduce that

$$
X=S_{u}^{1 / 2}\left(I+S_{u}^{1 / 2}\right)^{-1} K_{0},
$$

which implies condition $S_{u}^{1 / 2} K_{0}=K_{0} S_{y}^{1 / 2}$.

It remains to ensure that this is indeed a controller, i.e., $\mathcal{H}(G, X)$ is stable. This introduces the additional condition

$$
\begin{aligned}
S_{u}^{1 / 2} K_{0}=K_{0} S_{y}^{1 / 2} & =\text { stable } \\
S_{y}^{1 / 2} G=G S_{u}^{1 / 2} & =\text { stable. }
\end{aligned}
$$

Thus, we have obtained the existence conditions that fully characterise $X$ : there exists stable $S_{y}^{1 / 2}, S_{u}^{1 / 2}$ such that the inverses $\left(I+S_{y}^{1 / 2}\right)^{-1}$ and $\left(I+S_{u}^{1 / 2}\right)^{-1}$ also exists while (29) and (30) is satisfied. Observe that by the positivity of the square roots the existence of the inverses is granted. Since these inverses are not necessarily stable, the resulting controller should not be stable either.

In the SISO case and for stable plant and controller the existence conditions boils down to the existence of the stable square root system $S_{y}^{1 / 2}$.

As an illustrative numerical example take

$$
G=\left[\begin{array}{cc}
0 & 1 \\
0 & -3
\end{array}\right], \quad K_{0}=\left[\begin{array}{ll}
1 & 0 \\
0 & 1
\end{array}\right] .
$$

After some computation, which is left out for brevity, one has

$S_{u}^{1 / 2}=S_{y}^{1 / 2}=\left[\begin{array}{ll}1 & 1 / 6 \\ 0 & 1 / 2\end{array}\right],\left(I+S_{y}^{1 / 2}\right)^{-1}=\left[\begin{array}{cc}1 / 2 & -1 / 18 \\ 0 & 2 / 3\end{array}\right]$,

which leads to

$$
X=\left[\begin{array}{cc}
1 / 2 & 1 / 18 \\
0 & 1 / 3
\end{array}\right]
$$


Taking $K_{0}=\left[\begin{array}{ll}1 & 1 \\ 0 & 1\end{array}\right]$, we obtain

$$
S_{y}^{1 / 2}=\left[\begin{array}{cc}
1 & 1 / 6 \\
0 & 1 / 2
\end{array}\right], \quad S_{u}^{1 / 2}=\left[\begin{array}{cc}
1 & -1 / 3 \\
0 & 1 / 2
\end{array}\right],
$$

and

$$
X=\left[\begin{array}{cc}
1 / 2 & 7 / 18 \\
0 & 1 / 3
\end{array}\right]
$$

However, taking $K_{0}=\left[\begin{array}{ll}0 & 1 \\ 1 & 1\end{array}\right]$, the matrix $S_{y}^{1 / 2}$ will be complex, while $S_{u}^{1 / 2}$ is real. Thus (29) and (30) is violated, hence $X$ does not exist.

In a similar fashion one can obtain the existence conditions for the general case, i.e., for $X=\left[\frac{1}{n} K_{0}\right]_{\varpi_{G}}$ :

$$
K_{0}=X S_{y, X}^{-(k-1)}\left(\sum_{l=0}^{k-1} S_{y, X}^{l}\right),
$$

i.e.,

$$
X=K_{0}\left(\sum_{l=0}^{k-1} S_{y}^{\frac{l}{k}}\right)^{-1} S_{y}^{\frac{k-1}{k}}=\left(\sum_{l=0}^{k-1} S_{u}^{\frac{l}{k}}\right)^{-1} S_{u}^{\frac{k-1}{k}} K_{0} .
$$

If one would be tempted to replace the sums according to the identity $\sum_{l=0}^{k-1} Z^{l}=\left(I-Z^{k}\right)(I-Z)^{-1}$, note that $(I-$ $Z)^{-1}$ does not necessarily exists! Observe also that if $k>2$, having stable expressions $S_{u}^{1 / k} K_{0}=K_{0} S_{y}^{1 / k}$ and $S_{y}^{1 / k} G=$ $G S_{u}^{1 / k}$ are only sufficient to obtain a stabilizing $X$.

Apart from the trivial case when $S_{u}$ and $S_{y}$ are contractions it is less known how to ensure the existence of the required roots. Concerning the existence of the square root the only result found in the literature is the following: if the finite rank LTV systems $H$ and $(I+k H)^{-1}$ are both stable for all $0 \leq k \leq 1$ then, based on a Newton-Raphson technique of type

$$
Y_{i+1}=\frac{1}{2}\left((I+H) Y_{i}^{-1}+Y_{i}\right),
$$

one can show that $I+H$ has a stable square root, see [1], [2].

\section{Stabilization of the $P-K$ Loop}

Stability questions of LFT loops can be reduced to the investigation of the configuration determined by $(P, \bar{K})$, where $\bar{K}=\operatorname{diag}(0, K)$. It is obvious that the LFT loop is well-defined if and only if $(G, K)$ is well defined. However, it is less obvious whether this claim remains true for stability.

\section{A. Geometry of the LFT loop}

We already know that if $\left(P, \bar{K}_{1}\right)$ and $\left(P, \bar{K}_{2}\right)$ is stable, then $(P, \bar{K})$ is also stable with

$$
\bar{K}=\bar{K}_{1} \bullet_{P} \bar{K}_{2} .
$$

It is immediate to verify, that

$$
\begin{aligned}
\bar{K} & =\bar{K}_{1} \unrhd_{P} \bar{K}_{2}=\bar{K}_{1}+\bar{K}_{2}-\bar{K}_{1} P \bar{K}_{2}= \\
& =\operatorname{diag}\left(0, K_{1}+K_{2}-K_{1} G K_{2}\right)=\operatorname{diag}\left(0, K_{1} \triangleright_{G} K_{2}\right),
\end{aligned}
$$

i.e., $K=K_{1} \unlhd_{G} K_{2}$. Thus, well-definedness and stability of LFT loops is also a geometric property.

Moreover, a routine computation reveals that analogously to $(21)$, we have

$$
\mathcal{L}_{P, \bar{K}}=\mathcal{L}_{P, \bar{K}_{0}}-\left(\begin{array}{c}
S_{u} \\
S_{g} \\
-P_{z u} S_{u}
\end{array}\right) R\left(\begin{array}{lll}
S_{g} & S_{y} & -S_{y} P_{y w}
\end{array}\right),
$$

where the parameters correspond to $\left(G, K_{0}\right)$.

Thus, if $\left(P, \bar{K}_{0}\right)$ is stable, by using the blending rule (23) and observing that $\operatorname{diag}\left(0, \mathbb{Q}_{\Sigma}\right) \subset \mathbb{R}_{\bar{K}_{0}}$ we know by start a significant part of the stabilizing controllers for the LFT loop. Moreover, if we denote by $\mathbb{K}_{K_{0}}$ the set of controllers generated by using (23) and $\mathbb{Q}_{\Sigma}$, then these controllers will stabilize the LFT loop, too. It is an interesting research question whether we can infer from this that the LFT loop is stabilized by exactly those controllers that stabilize $G$.

When $G$ has a double coprime factorization, the answer is affirmative, as it will be shown in the next section.

B. $(G, K)$ vs. $(P, \bar{K})$ stability

One can easily check that

$$
\left(\begin{array}{cc}
P_{z w} & P_{z u} \\
P_{y w} & G
\end{array}\right)=\left(\begin{array}{cc}
P_{z w} & P_{z u} \\
P_{y w} & 0
\end{array}\right) \star\left(\begin{array}{cc}
0 & I \\
I & G
\end{array}\right) .
$$

Recall that the Redheffer star product is

$$
A \star B=\left(\begin{array}{cc}
\mathfrak{F}_{l}\left(A, B_{11}\right) & A_{12}\left(I-B_{11} A_{22}\right)^{-1} B_{12} \\
B_{21}\left(I-A_{22} B_{11}\right)^{-1} A_{21} & \mathfrak{F}_{u}\left(B, A_{22}\right)
\end{array}\right) .
$$

Thus, one has

$$
\begin{aligned}
& \mathfrak{F}_{l}(P, K)=\mathfrak{F}_{l}\left(\left(\begin{array}{cc}
P_{z w} & P_{z u} \\
P_{y w} & 0
\end{array}\right) \star\left(\begin{array}{cc}
0 & I \\
I & G
\end{array}\right), K\right)= \\
& =\mathfrak{F}_{l}\left(\left(\begin{array}{cc}
P_{z w} & P_{z u} \\
P_{y w} & 0
\end{array}\right), \mathfrak{F}_{l}\left(\left(\begin{array}{cc}
0 & I \\
I & G
\end{array}\right), K\right)\right)=\mathfrak{F}_{l}(\tilde{P}, \tilde{K}),
\end{aligned}
$$

where the map $\tilde{K}=K(I-G K)^{-1}$ has an inverse $K=$ $(I+\tilde{K} G)^{-1} \tilde{K}$ if and only if $\mathfrak{F}_{l}(P, K)$ is well defined.

From now on our assumption is that $G$ has a double coprime factorization, i.e., $G=n m^{-1}=\tilde{m}^{-1} \tilde{n}$ and

$\left(\begin{array}{cc}\tilde{v} & -\tilde{u} \\ -\tilde{n} & \tilde{m}\end{array}\right)\left(\begin{array}{cc}m & u \\ n & v\end{array}\right)=\left(\begin{array}{cc}m & u \\ n & v\end{array}\right)\left(\begin{array}{cc}\tilde{v} & -\tilde{u} \\ -\tilde{n} & \tilde{m}\end{array}\right)=\left(\begin{array}{ll}I & 0 \\ 0 & I\end{array}\right)$.

It follows then that the controllers $k$ that make the pair $(G, k)$ stable are described by the Youla parametrization

$$
k=(u+m q)(v+n q)^{-1}=(\tilde{v}+q \tilde{n})^{-1}(\tilde{u}+q \tilde{m})
$$

for arbitrary stable $q$ for which the corresponding inverses exists. We use the notation $k=u v^{-1}=\tilde{v}^{-1} \tilde{u}$ for the controller in order to emphasize our initial knowledge, i.e., the fact that the pair $(G, k)$ is stable. What we would like to prove is that $k$ also stabilizes the LFT loop, i.e., the pair $(P, \bar{K})$ is also stable with $\bar{K}=\left(\begin{array}{ll}0 & 0 \\ 0 & k\end{array}\right)$.

Our first observation is that by interchanging the role of $P$ and $\bar{K}$, we also have a double coprime factorization of the 
controllers $\bar{K}$ which makes the pair $(P, \bar{K})$ stable. Indeed, a possible factorization is given by

$$
\left(\begin{array}{cc|cc}
I & 0 & 0 & 0 \\
0 & \tilde{v} & 0 & -\tilde{u} \\
\hline 0 & 0 & I & 0 \\
0 & -\tilde{n} & 0 & \tilde{m}
\end{array}\right)\left(\begin{array}{cc|cc}
I & 0 & 0 & 0 \\
0 & m & 0 & u \\
\hline 0 & 0 & I & 0 \\
0 & n & 0 & v
\end{array}\right)=\left(\begin{array}{cc}
I & 0 \\
0 & I
\end{array}\right),
$$

i.e.,

$$
\left(\begin{array}{cc}
\tilde{V}_{0} & -\tilde{U}_{0} \\
-\tilde{N}_{0} & \tilde{M}_{0}
\end{array}\right)\left(\begin{array}{cc}
M_{0} & U_{0} \\
N_{0} & V_{0}
\end{array}\right)=\left(\begin{array}{ll}
I & 0 \\
0 & I
\end{array}\right) .
$$

Accordingly, we have all possible plants that makes the pair $\left(P_{K}, \bar{K}\right)$ stable as:

$$
\begin{aligned}
& P_{K}=\left(N_{0}+V_{0} Q\right)\left(M_{0}+U_{0} Q\right)^{-1}= \\
& =\left(\begin{array}{cc}
q_{11} & q_{12} \\
v q_{21} & n+v q_{22}
\end{array}\right)\left(\begin{array}{cc}
I & 0 \\
u q_{21} & m+u q_{22}
\end{array}\right)^{-1} .
\end{aligned}
$$

It follows that $P_{K}(2,2)=\left(n+v q_{22}\right)\left(m+u q_{22}\right)^{-1}$. Thus for $P_{K}(2,2)=G$ we have $q_{22}=0$ and

$$
\begin{aligned}
P_{K} & =\left(\begin{array}{cc}
q_{11} & q_{12} \\
v q_{21} & n
\end{array}\right)\left(\begin{array}{cc}
I & 0 \\
u q_{21} & m
\end{array}\right)^{-1}= \\
& =\left(\begin{array}{cc}
q_{11}-q_{12} m^{-1} u q_{21} & q_{12} m^{-1} \\
\tilde{m}^{-1} q_{21} & G
\end{array}\right) .
\end{aligned}
$$

It is easy to see that $\mathfrak{F}_{l}\left(P_{K}, K\right)=q_{11}$.

Thus,

$$
\begin{aligned}
& \left(\begin{array}{cc}
P_{z w} & P_{z u} \\
P_{y w} & 0
\end{array}\right)=\left(\begin{array}{cc}
q_{11}-q_{12} m^{-1} u q_{21} & q_{12} m^{-1} \\
\tilde{m}^{-1} q_{21} & 0
\end{array}\right)= \\
& =\left(\begin{array}{cc}
q_{11} & q_{12} \\
q_{21} & 0
\end{array}\right) \star\left(\begin{array}{cc}
-m^{-1} u & m^{-1} \\
\tilde{m}^{-1} & 0
\end{array}\right),
\end{aligned}
$$

and, for the given double coprime factorization of $G$, the stabilizable plants $P_{K}$ are given by

$$
P_{K}=\left(\begin{array}{cc}
q_{11} & q_{12} \\
q_{21} & 0
\end{array}\right) \star\left(\begin{array}{cc}
-m^{-1} u & m^{-1} \\
\tilde{m}^{-1} & 0
\end{array}\right) \star\left(\begin{array}{cc}
0 & I \\
I & G
\end{array}\right),
$$

where $q_{11}, q_{12}, q_{21}$ are stable systems.

Moreover,

$$
\begin{aligned}
& \mathfrak{F}_{l}\left(P_{K}, k\right)=\mathfrak{F}_{l}\left(\left(\begin{array}{cc}
P_{z w} & P_{z u} \\
P_{y w} & 0
\end{array}\right), k(I-G k)^{-1}\right)= \\
& =\mathfrak{F}_{l}\left(\left(\begin{array}{cc}
q_{11} & q_{12} \\
q_{21} & 0
\end{array}\right) \star\left(\begin{array}{cc}
-m^{-1} u & m^{-1} \\
\tilde{m}^{-1} & 0
\end{array}\right),(u+m q) \tilde{m}\right)= \\
& =\mathfrak{F}_{l}\left(\left(\begin{array}{cc}
q_{11} & q_{12} \\
q_{21} & 0
\end{array}\right), q\right)=q_{11}+q_{12} q q_{21} .
\end{aligned}
$$

According to (2) we should check that also $S_{c, k} P_{y w}$, $S_{y, k} P_{y w} P_{z u} S_{u, k}$ and $P_{y u} S_{c, k}$ is stable for the stabilizing controller $k$ given by (35). We have

$$
\begin{aligned}
& S_{c, k} P_{y w}=(u+m q) \tilde{m} \tilde{m}^{-1} q_{21}=(u+m q) q_{21}, \\
& S_{y, k} P_{y w}=(v+n q) \tilde{m} \tilde{m}^{-1} q_{21}=\left(v+n q q_{21},\right. \\
& P_{z u} S_{u, k}=q_{12} m^{-1} m(\tilde{v}+q \tilde{n})=q_{12}(\tilde{v}+q \tilde{n}), \\
& P_{z u} S_{c, k}=q_{12} m^{-1} m(\tilde{u}+q \tilde{m})=q_{12}(\tilde{u}+q \tilde{m}) .
\end{aligned}
$$

We are in a position to summarize stabilizability condition of (lower) LFT loops: the stabilizing controller set of an LFT loop coincides with the set of all stabilizing controllers $k$ of
$G$, and the closed-loop for a stabilizing controller is given by

$$
\mathfrak{F}_{l}(P, k)=q_{1}+q_{2} q q_{3},
$$

where $q$ is the Youla parameter of $K$ relative to the given double coprime factorization of $G$ and $q_{1}, q_{2}, q_{3}$ are stable systems.

Note that in this proof all the ingredients of the Youla parametrization play a decisive role. There is no way to repeat the argument if the parameter is not stable. Moreover, in this argument the stabilizability issue and the model matching property are strongly tight together.

\section{CONCLUSiOnS}

In this paper we provide conditions for the existence of fractional controllers relative to the stability preserving blending rule. We also extend our geometry based framework to the LFT loops. When double coprime factorization esists, it is shown that every controller which stabilizes the interior loop also stabilizes the LFT loop. Since existence of a stable stabilizing controller implies double coprime factorization, and in the LTV class always exists such a controller, this property holds for the interesting modell classes.

\section{REFERENCES}

[1] B. D. O. Anderson and S. Dasgupta, "Multiplier theory and operator square roots: Application to robust and time-varying stability," in Stability Theory, R. Jeltsch and M. Mansour, Eds. Basel: Birkhäuser Basel, pp. 113-124, 1996.

[2] S. Dasgupta and B. D. O. Anderson, "The square root of linear timevarying systems with applications," IEEE Trans. Circuits and Systems, vol. 43, no. 12, pp. 973-986, 1996.

[3] A. Feintuch, Robust Control Theory in Hilbert Space, ser. Applied Math. Sciences 130. Springer, New York, 1998.

[4] Gündes, A. N., Özbay, H.: Strong Stabilization of a Class of MIMO Systems. IEEE Transactions on Automatic Control, vol. 56, no. 6, 1445-1452, 2011.

[5] Kevickzy, L., Bányász, Cs.: Two-Degree-of-Freedom control systems: the Youla parameterization approach. Academic Press, 2015.

[6] P. P. Khargonekar, A. M. Pascoal, and R. Ravi, "Strong, simultaneous, and reliable stabilization of finite-dimensional linear time-varying plants," IEEE Transactions on Automatic Control, vol. 33, no. 12, pp. 1158-1161, 1988.

[7] V. Kucera, "Stability of discrete linear feedback systems," in Proceedings of the 6th IFAC. World Congress, Boston, MA, USA, 1975, 1975.

[8] Z. Szabó, J. Bokor, and T. Vámos, "A hyperbolic view on robust control," in Proc. of the 19th World Congress of the International Federation of Automatic Control, Cape Town, South Africa, 2014.

[9] Z. Szabó and J. Bokor, "Controller blending - a geometric approach," in ECC 2015, Linz, Austria, 2015.

[10] — "Jordan algebra and control theory," in ECC 2016, Aalborg, Denmark, 2016.

[11] Z. Szabó, J. Bokor, and T. Vámos, "Feedback stabilization: from geometry to control," IFAC-PapersOnLine, vol. 50, no. 1, pp. 1798 1804, 2017, 20th IFAC World Congress.

[12] Z. Szabó and J. Bokor, "Transformations for linear parameter varying systems," in Joint 9th IFAC Symposium on Robust Control Design and 2nd IFAC Workshop on Linear Parameter Varying Systems Florianopolis, Brazil, 2018, pp. 264-270.

[13] D. C. Youla, J. J. Bongiorno, Jr., and C. N. Lu, "Single-loop feedbackstabilization of linear multivariable dynamical plants," Automatica, vol. 10, no. 2, pp. 159-173, 1974.

[14] D. C. Youla, H. A. Jabr, and J. J. Bongiorno, "Modern Wiener-Hopf design of optimal controllers: Part II: The multivariable case," IEEE Transactions on Automatic Control, vol. 21, no. 3, pp. 319-338, 1976.

[15] M. Vidyasagar, Control System Synthesis: A Factorization Approach. MIT Press, Cambridge, 1985. 